\title{
Anti-gout Medications and Risk of Cardiovascular Disease: A Nested Case-Control Study
}

\author{
Tsung-Ju Chuang ${ }^{1,2}$, Yu-Hsun Wang ${ }^{3}$, James Cheng-Chung Wei ${ }^{4,5,6 * t}$ and \\ Chih-Jung Yeh ${ }^{2 * t}$
}

${ }^{1}$ Division of Endocrinology and Metabolism, Department of Internal Medicine, Taichung Armed Forces General Hospital, Taichung, National Defense Medical Center, Taipei, Taiwan, ${ }^{2}$ School of Public Health, Chung Shan Medical University, Taichung, Taiwan, ${ }^{3}$ Department of Medical Research, Chung Shan Medical University Hospital, Taichung, Taiwan, ${ }^{4}$ Department of Allergy, Immunology and Rheumatology, Chung Shan Medical University Hospital, Taichung, Taiwan, ${ }^{5}$ Institute of Medicine, Chung Shan Medical University, Taichung, Taiwan, ${ }^{6}$ Graduate Institute of Integrated Medicine, China Medical University, Taichung, Taiwan

\section{OPEN ACCESS}

Edited by:

Cong-Qiu Chu,

Oregon Health and Science University,

United States

Reviewed by:

Hejian ZOU,

Fudan University, China

Bernadette Heizel Reyes,

University of the Philippines

Manila, Philippines

${ }^{*}$ Correspondence:

Chih-Jung Yeh

alexyeh@csmu.edu.tw James Cheng-Chung Wei

wei3228@gmail.com

†These authors have contributed equally to this work

Specialty section: This article was submitted to Rheumatology,

a section of the journal

Frontiers in Medicine

Received: 11 July 2021 Accepted: 12 August 2021 Published: 18 October 2021

Citation:

Chuang T-J, Wang Y-H, Wei JC-C and Yeh C-J (2021) Anti-gout Medications and Risk of Cardiovascular Disease: A Nested Case-Control Study. Front. Med. 8:739680.

doi: 10.3389/fmed.2021.739680
Introduction: Gout is the leading cause of inflammatory arthritis and is also correlated with multiple comorbidities, including cardiovascular disease (CVD), whose future risk can be lowered by urate-lowering therapy (ULT) in gout patients. It is, however, still not clear whether its effect is associated with the days of usage and the adherence rate of ULT.

Methods: Data were collected from Taiwan's National Health Insurance Research Database. The study period was from 1999/1/1 to 2013/12/31. In addition, patients with newly diagnosed gout from 2000 to 2012 and usage of antigout preparations (allopurinol or benzbromarone) within half a year among age $\geq 20$ years old were enrolled in the study. The outcome of interest is CVD. New diagnosis of CVD after half a year of diagnosis of gout was included in the CVD group. Moreover, conditional logistic regression was used to evaluate the odds ratio of CVD in relation to the days of usage and to the adherence rate of ULT after the adjustment for potentially confounding variables.

Results: A total of 3,706 gout patients with and without CVD have been included in the final analysis after a 1:1 propensity score that matched for age, sex, comorbidities, aspirin, and statin. The days of usage of allopurinol was $<180$ days and benzbromarone, in its turn, presupposed a higher risk of CVD. The adherence rate of allopurinol and benzbromarone at $\geq 0.7$ both have a lower CVD risk: allopurinol (adjusted OR: 0.66 95\% Cl: 0.46-0.96), benzbromarone (adjusted OR: 0.68 95\% Cl: 0.50-0.91). The subgroup analysis revealed an adherence rate of $\geq 0.7$ of ULT with a lower CVD was only found to be present in males and at age $<65$. Furthermore, the correlations were more pronounced in the ischemic heart disease subgroup than in the cerebrovascular disease group.

Conclusion: This study reveals that gout patients taking ULT (allopurinol and benzbromarone) with an adherence rate of $\geq 0.7$ are at a lower risk of developing CVD, especially with a younger age $(<65)$ and if they are male. On top of this, the benefit is more pronounced in ischemic heart disease. Despite further prospective trials needing to be warranted to confirm our findings, health care providers may, bearing these conclusions in mind, emphasize the importance of adherence to ULT in gout patients.

Keywords: gout, cardiovascular disease, drug adherence, uric acid, urate-lowering therapy (ULT) 


\section{INTRODUCTION}

Gout is the main cause of inflammatory arthritis, usually affecting men, and is characterized by intermittent and sudden onset of intense inflammation (1), and it is characterized by hyperuricemia, serum, or plasma uric acid concentration of $>6.8$ $\mathrm{mg} / \mathrm{dL}$, close to the dissolution limit of uric acid (2). Gout may present itself through different manifestations of diseases, such as recurrent episodes of inflammatory arthritis, chronic joint disease, accumulation of urate crystals in the form of stone deposits, and uric acid (UA) kidney stones (3). In addition, gout is also associated with various comorbidities such as diabetes, hypertension, chronic kidney disease, and cardiovascular disease (CVD) (4).

The treatment of gout includes the treatment of acute attacks and chronic phases.

In the acute phase, several types of anti-inflammatory drugs are effective, including colchicine, non-steroidal antiinflammatory drugs, systemic and intra-articular glucocorticoids, and biological agents that inhibit the action of interleukin- $1 \beta$ $(3,5)$.

In the chronic stage, long-term use of urate-lowering therapy (ULT) can prevent gout flare, tophi formation, and related comorbidities (6). ULT usually includes xanthine oxidase inhibitors and uricosuric drugs, used to make serum uric acid levels $<6 \mathrm{mg} / \mathrm{dL}$, which is significantly lower than the uric acid solubility limit (6-8). The ULT should be selected according to the patient's hepatic and renal functions and their comorbidities.

Common xanthine oxidase inhibitors include allopurinol and febuxostat, which reduce the synthesis of UA by inhibiting the activity of xanthine oxidase (2). In adults, the starting dose of allopurinol is $50-100 \mathrm{mg} / \mathrm{d}$, which can be increased up to a maximum dose of $600 \mathrm{mg} / \mathrm{d}$ until the UA target is reached. In addition, the recommended dose for chronic kidney disease (CKD) stages 3-4 is $50-100 \mathrm{mg} / \mathrm{d}$, and CKD stage five patients are contraindicated (9). On the other hand, febuxostat is a selective xanthine oxidase inhibitor. The initial dose is $20-40 \mathrm{mg} / \mathrm{d}$, and it can be titrated up to a maximum of $80 \mathrm{mg} / \mathrm{d}$ until it reaches the serum UA target, which proves that it is effective in patients with impaired renal function, because it is mainly cleared by the liver (10).

The uricosuric drugs include probenecid, sulfinpyrazone, and benzbromarone.

Probenecid can be started at $250 \mathrm{mg}$ twice daily and may be increased to a maximum of $2 \mathrm{~g} /$ day. In addition, probenecid should be avoided in patients with eGFR $<30 \mathrm{~mL} / \mathrm{min}(11,12)$. In its turn, sulfinpyrazone starts at a dose of $50 \mathrm{mg}$ per day and can be increased to the maximum effective dose $(800 \mathrm{mg} /$ day $)$ and should be avoided in patients with CKD or history of UA kidney stones (13). Finally, the starting dose of benzbromarone is $25-50 \mathrm{mg} / \mathrm{d}$ in adults and should be adjusted to $75 \mathrm{mg} / \mathrm{d}$ or 100 $\mathrm{mg} / \mathrm{d}$ according to the serum UA level and is contraindicated in patients with severe renal dysfunction or UA nephrolithiasis (14). Moreover, its greatest adverse reaction is hepatotoxicity (15).

CVD is the leading cause of death worldwide, which may be due to the increased incidence of chronic diseases such as hypertension, hyperlipidemia, obesity, and diabetes $(16,17)$.
Apart from the traditional risk factors, gout and hyperuricemia were also found to be associated with CVD and its mortality (17-19). Moreover, several studies investigated the associations between ULT and CVD risk and mortality. Chen et al. found that hyperuricemia patients who received ULT had significantly lower all-cause mortality compared to the matched hyperuricemia patients who did not receive ULT. However, the CVD mortality risk between ULT users and non-users was not statistically significant due to a small number of mortality events (20). In addition, recent large-sample analyses further confirm that gout patients receiving ULT had a lower risk of further CAD and stroke $(21,22)$. It is, however, still not known whether the usage days and adherence rates of ULT are correlated with further risk of CAD.

In the study, we have therefore enrolled gout patients from the Taiwan Nation Health Insurance Research Database (NHIRD) in order to investigate the correlations between ULT (allopurinol and benzbromarone) and risk of CVD in gout patients, specifically focusing on the days of usage and on the adherence rates according to age and gender.

\section{METHODS}

\section{Data Source}

The data were collected from the Longitudinal Health Insurance Database (LHID) 2000, which contains the clinical information of 1 million beneficiaries that were randomly selected from the NHIRD during the period ranging from 2000 to 2013 (23). The data of NHIRD was based on a single-payer governmentrun National Health Insurance program developed in Taiwan in 1995, which currently includes comprehensive health care data (hospitalization, emergency care, and medical visits) for almost all 23 million Taiwanese citizens, and the databases were used for research purposes (24). The International Statistical Classification of Diseases and Related Health Problems, 9th Revision, Clinical Modification (ICD-9-CM) is used for the physicians' diagnosis, and the data include patients' medical drug items, dosage, frequency of use, and the number of days. In addition, this information can be used to detect drug interactions and potential repeated medications when patients visit multiple hospitals. In its turn, the National Health Insurance (NHI) code corresponds to a code in the five-level Anatomical Therapeutic Chemical classification system, having been recommended by the World Health Organization for studies on drug utilization (25).

\section{Data Collection}

The study period was from $1999 / 1 / 1$ to $2013 / 12 / 31$. We identified 1 million people from the database, of which we selected, based on the ICD-9-CM code, 274 patients with newly diagnosed gout from 2000 to 2012 undergoing ULT that included allopurinol (NHI code: M04AA01) or benzbromarone (NHI code: M04AB03), who used it within half a year, and who were $\geq 20$ years of age, having excluded the subjects treated by probenecid and sulfinpyrazone due to the small number of cases. In this regard, subjects that were diagnosed with CVD (ICD-9$\mathrm{CM}=410-414,430-438$ ) before the date of first gout diagnosis were also excluded. 
Individuals were divided into two groups, the CVD group and the non-CVD group, the former including individuals newly diagnosed with CVD followed by either $\geq 3$ outpatient assessments or a single inpatient admission after more than half a year of diagnosis of gout. These two groups were, then, initially matched 1:1 according to age ( $\pm 2 \mathrm{y} / \mathrm{o})$, sex, and the diagnosis year of gout. After the initial match, these two groups also received a Propensity Score Matching (PSM) 1:1 according to age, sex, comorbidities, aspirin, and statin.

\section{Patient Subgroups}

While the age of the patients was defined on the index date, 0 represented female and 1 represented male in relation to the sex variable. Furthermore, baseline comorbidities, which were diagnosed from three outpatient or one inpatient assessment 1 year before CVD diagnosis, included hypertension (ICD-9$\mathrm{CM}=401-405)$, hyperlipidemia (ICD-9-CM = 272.0-272.4), diabetes $(\mathrm{DM})(\mathrm{ICD}-9-\mathrm{CM}=250)$, chronic liver disease (CLD; ICD-9-CM = 571), chronic obstructive pulmonary disease (COPD; ICD-9-CM = 491, 492, and 496), and autoimmune disease (AD; ICD-9-CM $=710,714$, and 720). Finally, the use of aspirin or statin more than 30 days from the gout date to index date (CVD) was included in the baseline characteristics.

\section{Statistical Analysis}

All analyses were performed using SAS version 9.1.3 for Windows (SAS Institute, Inc., Cary, NC) and $P$-values $<0.05$ were considered statistically significant. Conditional logistic regression was used to evaluate the odds ratio (OR) of CVD in relation to the different ULT after due adjustment for potential confounding variables (age, sex, hypertension, hyperlipidemia, CLD, DM, COPD, AD, aspirin, statin, allopurinol, and benzbromarone). In addition, we analyzed the risks of CAD and stroke by three cumulative durations of ULT ( $<90$ days, $90-180$ days, or $\geq 180$ days) and the adherence rate of therapy $(<0.3,0.3-0.6$, or $\geq 0.7)$ relative to no-use of ULT in order to assess the dose-response relationship.

Kaplan-Meier analysis was applied to evaluate the cumulative incidence of CVD in the subgroups and log-rank test was used to test for significance. The subgroup analyses were, moreover, performed according to age $(<65$ years and $\geq 65$ years), gender, and different CVD (ischemic heart disease or cerebrovascular disease). The absolute standardized differences (ASD) were analyzed in the before PSM and after PSM matched samples for baseline covariates comparing CVD to non-CVD subjects, and a standardized difference of $10 \%$ (or 0.1 ) ASD is equivalent to having a phi coefficient of 0.05 (indicating negligible correlation) for the correlation between the treatment group and the binary variable (26). Drug adherence rate was measured as the days of usage of ULT, which were divided according to the observational period.

\section{RESULTS}

As shown in the flowchart in Figure 1, patients with and without CVD who were selected from the LHID 2000 and included in the final analysis were matched 1:1 by age, gender, and by the year

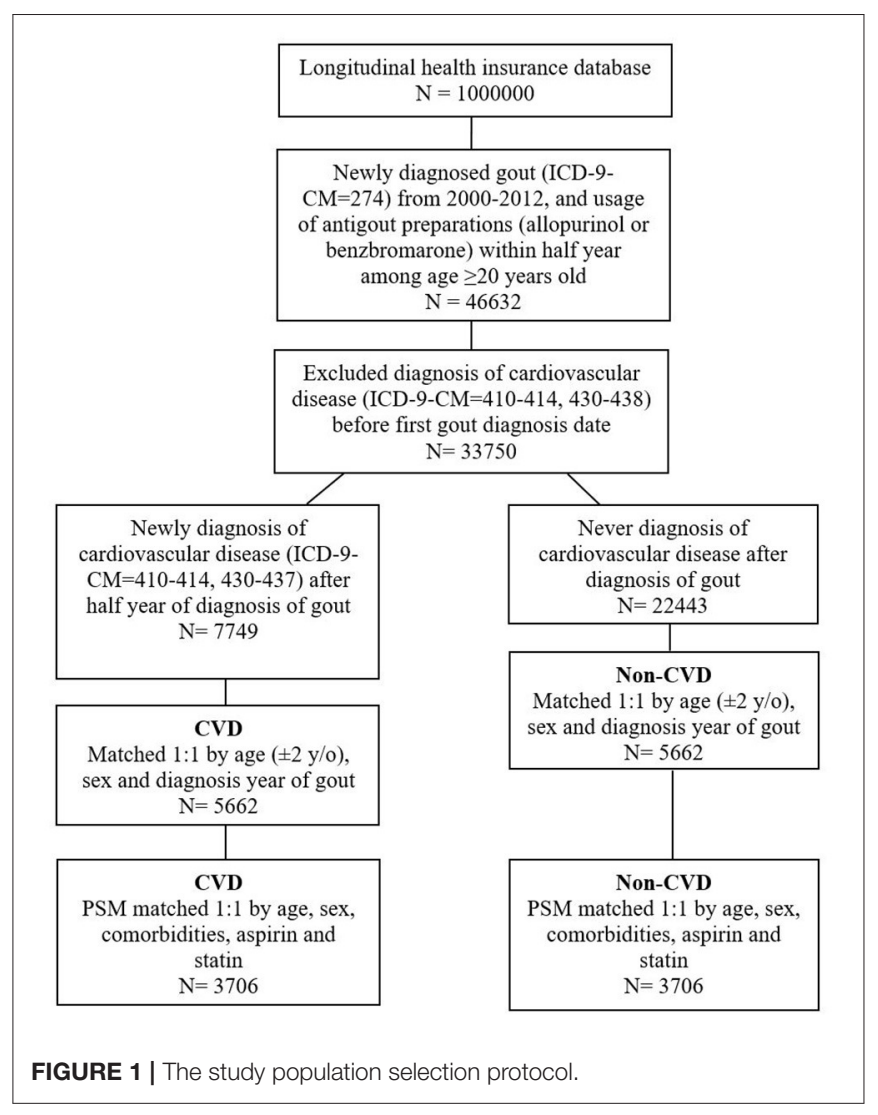

of diagnosis of gout, each group consisting of 5,662 patients, of which remained 3,706 patients in each after PSM 1:1 by age, sex, comorbidities, aspirin, and statin.

In this regard, Table 1 shows the demographic characteristics of the CVD and non-CVD groups before PSM and after PSM. Before PSM, only age, sex, autoimmune disease, allopurinol, and benzbromarone had an ASD lower than 0.1, whereas all the comorbidities, aspirin, and statin after PSM showed to have an ASD lower than 0.1, which indicated a negligible correlation.

Table 2 shows the risk of CVD divided according to days of usage of allopurinol and benzbromarone. In model 1, after adjusting for confounding factors (age, sex, hypertension, hyperlipidemia, CLD, DM, COPD, AD, and allopurinol or benzbromarone), fewer days of usage of allopurinol has a higher risk of CVD ( $<90$ days: OR, 1.18; 90-180 days: OR, 1.42) compared to the reference. In the benzbromarone group, fewer days of usage ( $<90$ days) likewise showed a higher risk of CVD $(\mathrm{OR}, 1.32)$. The trend of risks is shown in model 2 , being the same after adjusting for statin and aspirin.

In Table 3, the conditional logistic regression of risk of CVD was divided into the adherence rate of both allopurinol and benzbromarone $(<0.3,0.3-0.6, \geq 0.7)$, which, after adjusting for the confounding factors, statin and aspirin showed only an adherence rate of allopurinol and benzbromarone of $\geq 0.7$ and had a lower risk of CVD (allopurinol: adjusted OR, 0.66, $P: 0.03$; benzbromarone: adjusted OR, $0.68, P: 0.01$ ). 
TABLE 1 | Demographic characteristics.

\begin{tabular}{|c|c|c|c|c|c|c|c|c|c|c|}
\hline & \multicolumn{4}{|c|}{ Before PSM matched } & \multirow[b]{3}{*}{ ASD } & \multicolumn{4}{|c|}{ After PSM matched } & \multirow[b]{3}{*}{ ASD } \\
\hline & \multicolumn{2}{|c|}{ CVD $(N=5,662)$} & \multicolumn{2}{|c|}{ Non-CVD $(N=5,662)$} & & \multicolumn{2}{|c|}{ CVD $(N=3,706)$} & \multicolumn{2}{|c|}{ Non-CVD $(N=3,706)$} & \\
\hline & $n$ & $\%$ & $N$ & $\%$ & & $n$ & $\%$ & $N$ & $\%$ & \\
\hline Age & & & & & 0.067 & & & & & 0.050 \\
\hline $20-40$ & 340 & 6.0 & 351 & 6.2 & & 217 & 5.9 & 271 & 7.3 & \\
\hline $40-65$ & 3,607 & 63.7 & 3,799 & 67.1 & & 2,333 & 63.0 & 2,247 & 60.6 & \\
\hline$\geq 65$ & 1,715 & 30.3 & 1,512 & 26.7 & & 1,156 & 31.2 & 1,188 & 32.1 & \\
\hline Mean \pm SD & \multicolumn{2}{|c|}{$58.4 \pm 12$} & \multicolumn{2}{|c|}{$57.2 \pm 11.7$} & 0.101 & \multicolumn{2}{|c|}{$58.5 \pm 12.2$} & \multicolumn{2}{|c|}{$58.2 \pm 12.3$} & 0.021 \\
\hline Sex & & & & & 0.000 & & & & & \\
\hline Female & 1,527 & 27.0 & 1,527 & 27.0 & & 1,001 & 27.0 & 1,014 & 27.4 & \\
\hline Male & 4,135 & 73.0 & 4,135 & 73.0 & & 2,705 & 73.0 & 2,692 & 72.6 & \\
\hline Hypertension & 3,175 & 56.1 & 1,507 & 26.6 & 0.627 & 1,483 & 40.0 & 1,507 & 40.7 & 0.013 \\
\hline Hyperlipidemia & 1,377 & 24.3 & 705 & 12.5 & 0.310 & 698 & 18.8 & 681 & 18.4 & 0.012 \\
\hline Chronic liver disease & 470 & 8.3 & 303 & 5.4 & 0.117 & 256 & 6.9 & 275 & 7.4 & 0.020 \\
\hline Diabetes & 1,120 & 19.8 & 575 & 10.2 & 0.272 & 563 & 15.2 & 552 & 14.9 & 0.008 \\
\hline COPD & 245 & 4.3 & 113 & 2.0 & 0.134 & 95 & 2.6 & 112 & 3.0 & 0.028 \\
\hline Autoimmune disease & 95 & 1.7 & 68 & 1.2 & 0.040 & 45 & 1.2 & 51 & 1.4 & 0.014 \\
\hline Statin & 1,393 & 24.6 & 889 & 15.7 & 0.223 & 786 & 21.2 & 776 & 20.9 & 0.007 \\
\hline Aspirin & 1,447 & 25.6 & 399 & 7.0 & 0.518 & 435 & 11.7 & 399 & 10.8 & 0.031 \\
\hline Allopurinol & 2,620 & 46.3 & 2,453 & 43.3 & 0.059 & 1,681 & 45.4 & 1,630 & 44.0 & 0.028 \\
\hline Benzbromarone & 4,581 & 80.9 & 4,453 & 78.6 & 0.056 & 3,008 & 81.2 & 2,954 & 79.7 & 0.037 \\
\hline
\end{tabular}

ASD, Absolute standardized difference; COPD, Chronic obstructive pulmonary disease.

TABLE 2 | Logistic regression of risk of CVD divided by usage days of allopurinol and benzbromarone.

\begin{tabular}{|c|c|c|c|c|c|c|c|c|}
\hline & \multirow[t]{2}{*}{$N$} & \multirow[t]{2}{*}{ No. of CVD } & \multirow[t]{2}{*}{ cOR (95\% C.I.) } & \multirow[t]{2}{*}{$p$-value } & \multicolumn{2}{|c|}{ Model 1} & \multicolumn{2}{|c|}{ Model 2} \\
\hline & & & & & aOR (95\% C.I.) & $p$-value & aOR (95\% C.I.) & $p$-value \\
\hline \multicolumn{9}{|l|}{ Allopurinol } \\
\hline None & 4,101 & 2,025 & Reference & & Reference & & Reference & \\
\hline$<90$ days & 2,193 & 1,113 & 1.06 (0.95-1.17) & 0.299 & $1.18(1.05-1.34)$ & 0.008 & $1.18(1.04-1.34)$ & 0.008 \\
\hline 90-180 days & 427 & 237 & $1.28(1.05-1.56)$ & 0.016 & $1.42(1.15-1.75)$ & 0.001 & $1.42(1.15-1.74)$ & 0.001 \\
\hline$\geq 180$ days & 691 & 331 & $0.94(0.80-1.11)$ & 0.473 & $1.05(0.88-1.24)$ & 0.606 & $1.04(0.87-1.23)$ & 0.675 \\
\hline \multicolumn{9}{|l|}{ Benzbromarone } \\
\hline None & 1,450 & 698 & Reference & & Reference & & Reference & \\
\hline$<90$ days & 3,385 & 1,764 & $1.17(1.04-1.33)$ & 0.011 & $1.32(1.14-1.53)$ & $<0.001$ & $1.32(1.14-1.53)$ & $<0.001$ \\
\hline 90-180 days & 1,013 & 505 & $1.07(0.91-1.26)$ & 0.402 & $1.18(0.99-1.41)$ & 0.062 & $1.18(0.98-, 1.41)$ & 0.074 \\
\hline$\geq 180$ days & 1,564 & 739 & $0.97(0.84-1.11)$ & 0.626 & $1.05(0.89-1.23)$ & 0.546 & $1.04(0.89-1.22)$ & 0.622 \\
\hline
\end{tabular}

cOR, crude odds ratio; aOR, adjusted odds ratio.

Model 1: Adjusted for allopurinol, benzbromarone, age, sex, hypertension, hyperlipidemia, chronic liver disease, diabetes, COPD, and autoimmune disease.

Model 2: Adjusted for allopurinol, benzbromarone, age, sex, hypertension, hyperlipidemia, chronic liver disease, diabetes, COPD, autoimmune disease, statin, and aspirin.

In Table 4, the adherence rate of allopurinol and benzbromarone was further divided in relation to age $(<65$, $\geq 65$ years) and gender. Evidently, the logistic regression of risk of CVD in the younger group ( $<65$ years) showed lower risks with the increased adherence of ULT, of which a rate of $\geq 0.7$ had lower CVD risk in both allopurinol (adjusted OR, 0.62; 95\% CI, 0.38-1.0) and benzbromarone (adjusted OR, 0.61; 95\% CI, 0.41-0.90). All adherence rates of both allopurinol and benzbromarone, however, did not have a significantly lower risk of CVD in patients $\geq 65$ years. In relation to gender, the larger adherence rate of both allopurinol and benzbromarone showed a lower risk of CVD in males as opposed to females. Specifically, the adjusted OR is 0.63 in allopurinol and 0.64 in benzbromarone with an adherence rate of $\geq 0.7$.

In Table 5, the CVD were further divided into ischemic heart disease and cerebrovascular disease. Regarding the former, allopurinol and benzbromarone both had a higher risk of ischemic heart disease with an adherence rate of $<0.3$ and 
TABLE 3 | Logistic regression of risk of CVD divided by adherence rate of allopurinol and benzbromarone.

\begin{tabular}{|c|c|c|c|c|c|c|}
\hline & $N$ & No. of CVD & Crude OR (95\% C.I.) & $p$-value & Adjusted $\mathrm{OR}^{\dagger}(95 \%$ C.I.) & $p$-value \\
\hline \multicolumn{7}{|c|}{ Adherence rate of allopurinol } \\
\hline None & 4,101 & 2,025 & Reference & & Reference & \\
\hline$<0.3$ & 2,908 & 1,505 & $1.10(1.00-1.21)$ & 0.05004 & $1.15(1.03-1.28)$ & 0.017 \\
\hline $0.3-0.6$ & 266 & 125 & $0.91(0.71-1.17)$ & 0.451 & $0.95(0.73-1.23)$ & 0.702 \\
\hline$\geq 0.7$ & 137 & 51 & $0.61(0.43-0.86)$ & 0.006 & $0.66(0.46-0.96)$ & 0.030 \\
\hline$<0.3$ & 5,072 & 2,611 & $1.14(1.02-1.28)$ & 0.025 & $1.19(1.03-1.37)$ & 0.015 \\
\hline $0.3-0.6$ & 647 & 306 & $0.97(0.80-1.16)$ & 0.721 & $1.00(0.82-1.23)$ & 0.975 \\
\hline$\geq 0.7$ & 243 & 91 & $0.65(0.49-0.85)$ & 0.002 & $0.68(0.50-0.91)$ & 0.010 \\
\hline
\end{tabular}

${ }^{\dagger}$ Adjusted for allopurinol, benzbromarone, age, sex, hypertension, hyperlipidemia, chronic liver disease, diabetes, COPD, autoimmune disease, statin, and aspirin.

a lower risk with an adherence rate of $>0.7$. In the group of cerebrovascular disease, however, only an adherence rate of benzbromarone of $>0.7$ proved to decrease the risk of cerebrovascular disease ( $p$-value: 0.028$)$.

\section{DISCUSSIONS}

Our study showed that only a high adherence rate of receiving ULT (allopurinol and benzbromarone) in gout patients was associated with lower risks of future CVD. In their turn, the subgroup analyses were able to reveal the trend is particularly significant in male patients and those of a younger age ( $<65$ years).

The common use of ULT for gout patients is different according to each country.

A nationwide population study conducted from 2005 to 2010 in Taiwan reported that the prevalence of gout may be as high as $6.24 \%$. However, only $22.93 \%$ of patients received ULT (27), among which $60.08 \%$ received uricosuric agents only (mostly benzbromarone), $28.54 \%$ received allopurinol, and $11.38 \%$ received both. In the present study, we have enrolled gout patients from 2000 to 2012, and like the previous epidemiology study, most patients use benzbromarone and Allopurinol for ULT. Additionally, the other recently commonly used xanthine oxidase inhibitor is febuxostat, which was approved by the U.S. Food and Drug Administration in 2009 and was not listed in Taiwan until 2012. Fewer patients in the study period used this drug and cannot, therefore, be enrolled in the analysis.

Previous studies have reported that elevated serum UA is closely related to coronary and carotid atherosclerosis $(21,28)$. Specifically, Mutluay et al. have reported that a higher serum UA in hypertensive subjects with normal renal function is an independent predictor of early atherosclerosis (29). The mechanisms linking elevated serum UA levels and gout to CVD comorbidities appear to be multifactorial, involving lowgrade systemic inflammation and xanthine oxidase activity. Jacob George et al. have reported that UA acts as a pro-oxidant and induces endothelial dysfunction (30). In contrast, UA may also stimulate the renin-angiotensin system to further promote the growth of smooth blood vessel cells, as well as impaired arterial function and stiffening (31). Moreover, the correlation between hyperuricemia and systemic inflammatory markers (such as Creactive protein) and tumor necrosis was also explored, showing a further contribution to CV damage (31); use of ULT may lower, as expected, serum UA, decreasing the risk of CVD. Hence, a past study has found that gout patients receiving ULT had a $32 \%$ lower risk of CVD compared with those not receiving ULT (22).

Dubreuil et al. have reported allopurinol can reduce allcause and CVD mortality in the general population (32). Being treated with allopurinol over a 10-year period can reduce the risk of stroke $(-50 \%)$ and cardiac event $(-39 \%)$ in hypertensive patients, this being particularly significant in patients treated with a higher allopurinol dose (33). The dose-dependent trend was also found by Yen et al. their subgroup analyses indicating uricosuric agents among the ULT had a significant effect on the prevention of CVD development (21).

In the present study, we were further able to find that, instead, fewer days of usage of ULT (allopurinol $<180$ days and benzbromarone $<90$ days) increase the risks of CVD compared to the reference in gout patients, which can further emphasize the importance of the dose-dependent effect of ULT on CVD. The compliance and the adherence rate of ULT is important in gout patients and can influence the risk of CVD.

Likewise, a systematic review reported that less than half of gout patients in the real world adhere to treatment, and healthcare professionals in medication may emphasize the importance of monitoring, discussing, and supporting adherence to ULT $(34,35)$. The association between the adherence rate and the risk of CVD has not, however, been analyzed in the past, and we were able to find the adherence rate of ULT may significantly influence the risk of CVD in gout patients, in which only an adherence rate of $\geq 0.7$ is able to reduce the risk of CVD both in the allopurinol (adjusted OR, 0.66) and benzbromarone (adjusted OR, 0.68) groups (Table 3).

The subgroup analysis revealed the protective effect of ULT on CVD was only seen in male patients and those of a younger 
TABLE 4 | Logistic regression of risk of CVD divided by adherence rate of allopurinol and benzbromarone in different age and gender groups.

\begin{tabular}{|c|c|c|c|c|c|c|}
\hline & $\boldsymbol{N}$ & No. of CVD & Crude OR (95\% C.I.) & $p$-value & Adjusted OR ${ }^{\dagger}$ (95\% C.I.) & $p$-value \\
\hline \multicolumn{7}{|l|}{ Age $<65$} \\
\hline \multicolumn{7}{|c|}{ Adherence rate of allopurinol } \\
\hline None & 2,749 & 1,382 & Reference & & Reference & \\
\hline$<0.3$ & 2,064 & 1,064 & $1.05(0.94-1.18)$ & 0.38028 & $1.11(0.97-1.26)$ & 0.140 \\
\hline $0.3-0.6$ & 174 & 74 & $0.73(0.54-1.00)$ & 0.048 & $0.77(0.55-1.06)$ & 0.104 \\
\hline None & 982 & 466 & Reference & & Reference & \\
\hline$<0.3$ & 3,547 & 1,837 & $1.19(1.03-1.37)$ & 0.016 & $1.20(1.01-1.42)$ & 0.037 \\
\hline $0.3-0.6$ & 401 & 197 & $1.07(0.85-1.35)$ & 0.572 & $1.05(0.81-1.35)$ & 0.707 \\
\hline$\geq 0.7$ & 138 & 50 & $0.63(0.44-0.91)$ & 0.014 & $0.61(0.41-0.90)$ & 0.013 \\
\hline \multicolumn{7}{|l|}{ Age $\geq 65$} \\
\hline $0.3-0.6$ & 92 & 51 & $1.37(0.90-2.10)$ & 0.145 & $1.45(0.93-2.25)$ & 0.099 \\
\hline$\geq 0.7$ & 56 & 21 & $0.66(0.38-1.15)$ & 0.142 & $0.72(0.40-1.29)$ & 0.270 \\
\hline \multicolumn{7}{|c|}{ Adherence rate of benzbromarone } \\
\hline None & 468 & 232 & Reference & & Reference & \\
\hline$<0.3$ & 1,525 & 774 & $1.05(0.85-1.29)$ & 0.655 & $1.17(0.91-1.51)$ & 0.218 \\
\hline $0.3-0.6$ & 246 & 109 & $0.81(0.59-1.10)$ & 0.181 & $0.94(0.66-1.34)$ & 0.729 \\
\hline$\geq 0.7$ & 105 & 41 & $0.65(0.42-1.00)$ & 0.052 & $0.78(0.49-1.24)$ & 0.294 \\
\hline \multicolumn{7}{|l|}{ Female } \\
\hline \multicolumn{7}{|c|}{ Adherence rate of allopurinol } \\
\hline None & 1,268 & 623 & Reference & & Reference & \\
\hline$<0.3$ & 653 & 336 & $1.10(0.91-1.33)$ & 0.335 & $1.16(0.91-1.48)$ & 0.220 \\
\hline$\geq 0.7$ & 69 & 27 & $0.67(0.40-1.13)$ & 0.133 & $0.77(0.44-1.35)$ & 0.362 \\
\hline \multicolumn{7}{|l|}{ Male } \\
\hline \multicolumn{7}{|c|}{ Adherence rate of allopurinol } \\
\hline None & 2,833 & 1,402 & Reference & & Reference & \\
\hline$<0.3$ & 2,255 & 1,169 & $1.10(0.98-1.23)$ & 0.096 & $1.14(1.01-1.30)$ & 0.040 \\
\hline $0.3-0.6$ & 210 & 98 & $0.89(0.67-1.18)$ & 0.430 & $0.92(0.69-1.24)$ & 0.589 \\
\hline$\geq 0.7$ & 99 & 36 & $0.58(0.38-0.88)$ & 0.011 & $0.63(0.41-0.97)$ & 0.037 \\
\hline \multicolumn{7}{|c|}{ Adherence rate of benzbromarone } \\
\hline None & 1,035 & 495 & Reference & & Reference & \\
\hline$<0.3$ & 3,733 & 1,931 & $1.17(1.02-1.34)$ & 0.026 & $1.20(1.02-1.41)$ & 0.024 \\
\hline $0.3-0.6$ & 455 & 215 & $0.98(0.78-1.22)$ & 0.838 & $0.98(0.77-1.25)$ & 0.893 \\
\hline$\geq 0.7$ & 174 & 64 & $0.63(0.46-0.88)$ & 0.007 & $0.64(0.45-0.91)$ & 0.013 \\
\hline
\end{tabular}

${ }^{\dagger}$ Adjusted for allopurinol, benzbromarone, age, sex, hypertension, hyperlipidemia, chronic liver disease, diabetes, COPD, autoimmune disease, statin, and aspirin.

age $(<65)$. We can also find the trend at an older age and in females, but it is not significant. In this regard, the epidemiology study showed that the prevalence of gout is significantly higher in men. In men, the overall prevalence of gout can be 2.9 -fold higher than in women, with this difference being observed in all ages and peaking at 7.3-fold from 30 to 34 years of age, showing to decrease thereafter (27). Gout in males is, therefore, an apparently younger onset disease; Li found that patients 
TABLE 5 | Sub-outcome of logistic regression of risk of CVD.

\begin{tabular}{|c|c|c|c|c|c|c|}
\hline & $\mathbf{N}$ & No. of CVD & Crude OR (95\% C.I.) & $p$-value & Adjusted $\mathrm{OR}^{\dagger}$ (95\% C.I.) & $p$-value \\
\hline \multicolumn{7}{|c|}{ Ischemic heart disease } \\
\hline \multicolumn{7}{|c|}{ Adherence rate of allopurinol } \\
\hline None & 2,527 & 451 & Reference & & Reference & \\
\hline$<0.3$ & 1,761 & 358 & $1.17(1.01-1.37)$ & 0.041 & $1.20(1.00-1.43)$ & 0.0497 \\
\hline $0.3-0.6$ & 170 & 29 & $0.95(0.63-1.43)$ & 0.795 & $1.02(0.66-1.56)$ & 0.930 \\
\hline$\geq 0.7$ & 93 & 7 & $0.37(0.17-0.81)$ & 0.013 & $0.46(0.21-1.02)$ & 0.057 \\
\hline \multicolumn{7}{|c|}{ Adherence rate of benzbromarone } \\
\hline None & 904 & 152 & Reference & & Reference & \\
\hline$<0.3$ & 3,076 & 615 & $1.24(1.02-1.50)$ & 0.033 & $1.29(1.02-1.62)$ & 0.030 \\
\hline $0.3-0.6$ & 405 & 64 & $0.93(0.68-1.28)$ & 0.649 & $1.02(0.72-1.45)$ & 0.891 \\
\hline$\geq 0.7$ & 166 & 14 & $0.46(0.26-0.81)$ & 0.007 & $0.51(0.28-0.93)$ & 0.028 \\
\hline \multicolumn{7}{|c|}{ Cerebrovascular disease } \\
\hline \multicolumn{7}{|c|}{ Adherence rate of allopurinol } \\
\hline None & 3,650 & 1,574 & Reference & & Reference & \\
\hline$<0.3$ & 2,550 & 1,147 & $1.08(0.97-1.19)$ & 0.147 & $1.12(0.99-1.26)$ & 0.061 \\
\hline $0.3-0.6$ & 237 & 96 & $0.90(0.69-1.17)$ & 0.431 & $0.93(0.70-1.22)$ & 0.593 \\
\hline$\geq 0.7$ & 130 & 44 & $0.67(0.47-0.98)$ & 0.037 & $0.71(0.48-1.05)$ & 0.083 \\
\hline \multicolumn{7}{|c|}{ Adherence rate of benzbromarone } \\
\hline None & 1,298 & 546 & Reference & & Reference & \\
\hline$<0.3$ & 4,457 & 1,996 & $1.12(0.99-1.27)$ & 0.083 & $1.16(1.00-1.34)$ & 0.058 \\
\hline $0.3-0.6$ & 583 & 242 & $0.98(0.80-1.19)$ & 0.821 & $1.00(0.80-1.24)$ & 0.977 \\
\hline$\geq 0.7$ & 229 & 77 & $0.70(0.52-0.94)$ & 0.017 & $0.71(0.52-0.97)$ & 0.034 \\
\hline
\end{tabular}

${ }^{\dagger}$ Adjusted for allopurinol, benzbromarone, age, sex, hypertension, hyperlipidemia, chronic liver disease, diabetes, COPD, autoimmune disease, statin, and aspirin.

with a first diagnosis of gout at a younger age may be at an increased risk for CVD as opposed to those diagnosed later in life (36). This higher CVD risk in younger gout patients may explain the more significant effect in lowering CVD the higher adherence rate of ULT has in male patients and those of $<65$ years of age.

Moreover, the subgroup analysis also reported the high adherence to benzbromarone can reduce both incident ischemic heart disease and cerebrovascular disease (including ischemic and hemorrhagic stroke). However, the high adherence to allopurinol only have trend, proving not to play a significant role in reducing the risk of cerebrovascular disease. Apart from the fewer cases of allopurinol needing more subjects to achieve significance, the outcome was not totally consistent with the previous studies. Fu-Shun Yen et al. have reported ULT users among the patients with gout showed a lower risk of hospitalized ischemic stroke and all-cause mortality as opposed to non-users; the risks of hospitalized CAD, CV death, and hemorrhagic stroke do not, however, have obviously decrease (37). In its turn, FuShun Yen et. Al. have also found ULT to be correlated with lower risks of incident $\mathrm{CAD}$ and stroke, with the subgroup analysis also revealing the effect is only significant in ischemic stroke as opposed to hemorrhagic stroke (21). Further prospective trials, specifically regarding the ischemic stroke, are further warranted to confirm our findings.

\section{CONCLUSIONS}

The present study investigated the correlations between ULT (allopurinol and benzbromarone) and the risk of CVD in gout patients, showing that only a high adherence rate $(\geq 0.7)$ of ULT can significantly reduce the risk of CVD, especially at a younger age ( $<65$ years) and in male patients. Therefore, health care providers who prescribe gout medications may emphasize the importance of monitoring and supporting adherence to this therapy.

\section{DATA AVAILABILITY STATEMENT}

The original contributions presented in the study are included in the article/supplementary material, further inquiries can be directed to the corresponding author/s.

\section{ETHICS STATEMENT}

Ethical review and approval was not required for the study on human participants in accordance with the local legislation and institutional requirements. Written informed consent for participation was not required for this 
study in accordance with the national legislation and the institutional requirements.

\section{AUTHOR CONTRIBUTIONS}

All authors listed have made a substantial, direct and intellectual contribution to the work, and approved it for publication.

\section{REFERENCES}

1. Miao Z, Li C, Chen Y, Zhao S, Wang Y, Wang Z, et al. Dietary and lifestyle changes associated with high prevalence of hyperuricemia and gout in the Shandong coastal cities of Eastern China. J Rheumatol. (2008) 35:1859-64.

2. Loeb JN. The influence of temperature on the solubility of monosodium urate. Arthritis Rheum. (1972) 15:189-92. doi: 10.1002/art.1780150209

3. Richette P, Bardin T. Gout. Lancet. (2010) 375:31828. doi: 10.1016/S0140-6736(09)60883-7

4. Robinson PC, Merriman TR, Herbison P, Highton J. Hospital admissions associated with gout and their comorbidities in New Zealand and England 1999-2009. Rheumatology (Oxford). (2013) 52:118-26. doi: 10.1093/rheumatology/kes253

5. Sundy JS. Progress in the pharmacotherapy of gout. Curr Opin Rheumatol. (2010) 22:188-93. doi: 10.1097/BOR.0b013e3283369014

6. Qaseem A, Harris RP, Forciea MA. Management of acute and recurrent gout: a clinical practice guideline from the American College of Physicians. Ann Intern Med. (2017) 166:58-68. doi: 10.7326/M16-0570

7. Wallace SL, Singer JZ. Therapy in gout. Rheum Dis Clin North Am. (1988) 14:441-57. doi: 10.1016/S0889-857X(21)00975-3

8. Zhang W, Doherty M, Bardin T, Pascual E, Barskova V, Conaghan P, et al. EULAR evidence based recommendations for gout. Part II: Management Report of a task force of the EULAR Standing Committee for International Clinical Studies Including Therapeutics (ESCISIT). Ann Rheum Dis. (2006) 65:1312-24. doi: 10.1136/ard.2006.055269

9. Stamp LK, Taylor WJ, Jones PB, Dockerty JL, Drake J, Frampton C, et al. Starting dose is a risk factor for allopurinol hypersensitivity syndrome: a proposed safe starting dose of allopurinol. Arthritis Rheum. (2012) 64:252936. doi: 10.1002/art.34488

10. Schumacher HR. Jr. <jabb>, Becker MA, Wortmann RL, Macdonald PA, Hunt B, Streit J, et al. Effects of febuxostat versus allopurinol and placebo in reducing serum urate in subjects with hyperuricemia and gout: a 28 week, phase III, randomized, double-blind, parallel-group trial Arthritis Rheum. </jabb> (2008) 59:1540-8. doi: 10.1002/art.24209

11. Khanna D, Fitzgerald JD, Khanna PP, Bae S, Singh MK, Neogi T, et al. 2012 American College of Rheumatology guidelines for management of gout. Part 1: systematic nonpharmacologic and pharmacologic therapeutic approaches to hyperuricemia. Arthritis Care Res (Hoboken). (2012) 64:143146. doi: 10.1002/acr.21772

12. Mason RM. Studies on the Effect of Probenecid ('Benemid') in Gout. Ann Rheum Dis. (1954) 13:120-30. doi: 10.1136/ard.13.2.120

13. Underwood M. Diagnosis and management of gout. BMJ (Clinical research ed). (2006) 332:1315-9. doi: 10.1136/bmj.332.7553.1315

14. Fujimori S, Ooyama K, Ooyama H, Moromizato H. Efficacy of benzbromarone in hyperuricemic patients associated with chronic kidney disease. Nucleosides Nucleotides Nucleic Acids. (2011) 30:1035-8. doi: 10.1080/15257770.2011.622732

15. van Echteld IA, van Durme C, Falzon L, Landewé RB, van der Heijde DM, Aletaha D. Treatment of gout patients with impairment of renal function: a systematic literature review. J Rheumatol Suppl. (2014) 92:4854. doi: 10.3899/jrheum. 140462

\section{FUNDING}

This study was supported by the Taichung Armed Forces General Hospital (Grant No.: TCAFGH-D-109011).

\section{ACKNOWLEDGMENTS}

This study received assistance from the Department of Medical Education and Research, Taichung Armed Forces General Hospital.

16. Dhindsa DS, Khambhati J, Sandesara PB, Eapen DJ, Quyyumi AA. Biomarkers to predict cardiovascular death. Card Electrophysiol Clin. (2017) 9:65164. doi: 10.1016/j.ccep.2017.07.014

17. Mora-Ramírez M, Estevez-Garcia IO, Irigoyen-Camacho ME, Bojalil R, Gonzalez-Pacheco H, Amezcua-Guerra LM. Hyperuricemia on admission predicts short-term mortality due to myocardial infarction in a population with high prevalence of cardiovascular risk factors. Rev Invest Clin. (2017) 69:247-53. doi: 10.24875/RIC.17002167

18. Krishnan E. Gout and coronary artery disease: epidemiologic clues. Curr Rheumatol Rep. (2008) 10:249-55. doi: 10.1007/s11926-008-0039-0

19. Huang KH, Tai CJ, Tsai YF, Kuan YH, Lee CY. Correlation between gout and coronary heart disease in Taiwan: a nationwide population-based cohort study. Acta Cardiol Sin. (2019) 35:634-40.

20. Chen J-H, Lan J-L, Cheng C-F, Liang W-M, Lin H-Y, Tsay GJ, et al. Effect of urate-lowering therapy on all-cause and cardiovascular mortality in hyperuricemic patients without gout: a case-matched cohort study. PloS ONE. (2015) 10:e0145193-e. doi: 10.1371/journal.pone.0145193

21. Yen FS, Hsu CC Li HL, Wei JC, Hwu CM. Urate-lowering therapy may prevent the development of coronary artery disease in patients with gout. Front Med (Lausanne). (2020) 7:63. doi: 10.3389/fmed.2020.00063

22. Huang WS, Lin CL, Tsai CH, Chang KH. Association of gout with CAD and effect of antigout therapy on CVD risk among gout patients. I Investig Med. (2020) 68:972-9. doi: 10.1136/jim-2019-001140

23. Chen H-H, Perng W-T, Chiou J-Y, Wang Y-H, Huang J-Y, Wei JC-C. Risk of dementia among patients with Sjogren's syndrome: A nationwide population-based cohort study in Taiwan. Semin Arthritis Rheum. (2019) 48:895-9. doi: 10.1016/j.semarthrit.2018.06.007

24. Hsing AW, Ioannidis JPA. Nationwide population science: lessons from the Taiwan National Health Insurance Research Database. JAMA Intern Med. (2015) 175:1527-9. doi: 10.1001/jamainternmed.2015.3540

25. Hsu M-H, Yeh Y-T, Chen C-Y, Liu C-H, Liu C-T. Online detection of potential duplicate medications and changes of physician behavior for outpatients visiting multiple hospitals using national health insurance smart cards in Taiwan. Int J Med Inform. (2011) 80:181-9. doi: 10.1016/j.ijmedinf.2010.11.003

26. Austin PC. Using the standardized difference to compare the prevalence of a binary variable between two groups in observational research. Commun Stat-Simul Comput. (2009) 38:1228-34. doi: 10.1080/03610910902859574

27. Kuo C-F, Grainge MJ, See L-C, Yu K-H, Luo S-F, Zhang W, et al. Epidemiology and management of gout in Taiwan: a nationwide population study. Arthritis Res Ther. (2015) 17:13. doi: 10.1186/s13075-015-0522-8

28. Crouse JR, Toole JF, McKinney WM, Dignan MB, Howard G, Kahl FR, et al. Risk factors for extracranial carotid artery atherosclerosis. Stroke. (1987) 18:990-6. doi: 10.1161/01.STR.18.6.990

29. Mutluay R, Deger SM, Bahadir E, Durmaz AO, Citil R, Sindel S. Uric acid is an important predictor for hypertensive early atherosclerosis. Adv Ther. (2012) 29:276-86. doi: 10.1007/s12325-012-0006-z

30. George J, Carr E, Davies J, Belch JJ, Struthers A. High-dose allopurinol improves endothelial function by profoundly reducing vascular oxidative stress and not by lowering uric acid. Circulation. (2006) 114:250816. doi: 10.1161/CIRCULATIONAHA.106.651117 
31. Borghi C, Rosei EA, Bardin T, Dawson J, Dominiczak A, Kielstein JT, et al. Serum uric acid and the risk of cardiovascular and renal disease. J Hypertens. (2015) 33:1729-41. doi: 10.1097/HJH.00000000000 00701

32. Dubreuil M, Zhu Y, Zhang Y, Seeger JD, Lu N, Rho YH, et al. Allopurinol initiation and all-cause mortality in the general population. Ann Rheum Dis. (2015) 74:1368-72. doi: 10.1136/annrheumdis-2014-205269

33. MacIsaac RL, Salatzki J, Higgins P, Walters MR, Padmanabhan S, Dominiczak AF, et al. Allopurinol and cardiovascular outcomes in adults with hypertension. Hypertension. (2016) 67:535-40. doi: 10.1161/HYPERTENSIONAHA.115. 06344

34. De Vera MA, Marcotte G, Rai S, Galo JS, Bhole V. Medication adherence in gout: a systematic review. Arthritis Care Res (Hoboken). (2014) 66:15519. doi: 10.1002/acr.22336

35. Yin R, Li L, Zhang G, Cui Y, Zhang L, Zhang Q, et al. Rate of adherence to urate-lowering therapy among patients with gout: a systematic review and meta-analysis. BMJ Open. (2018) 8:e017542. doi: 10.1136/bmjopen-2017-017542

36. Li Y, Piranavan P, Sundaresan D, Yood R. Clinical characteristics of earlyonset gout in outpatient setting. ACR Open Rheumatol. (2019) 1:397402. doi: 10.1002/acr2.11057
37. Yen FS, Hsu CC Li HL, Wei JC, Hwu CM. Urate-lowering therapy may mitigate the risks of hospitalized stroke and mortality in patients with gout. PLoS ONE. (2020) 15:e0234909. doi: 10.1371/journal.pone.0234909

Conflict of Interest: The authors declare that the research was conducted in the absence of any commercial or financial relationships that could be construed as a potential conflict of interest.

Publisher's Note: All claims expressed in this article are solely those of the authors and do not necessarily represent those of their affiliated organizations, or those of the publisher, the editors and the reviewers. Any product that may be evaluated in this article, or claim that may be made by its manufacturer, is not guaranteed or endorsed by the publisher.

Copyright (c) 2021 Chuang, Wang, Wei and Yeh. This is an open-access article distributed under the terms of the Creative Commons Attribution License (CC BY). The use, distribution or reproduction in other forums is permitted, provided the original author(s) and the copyright owner(s) are credited and that the original publication in this journal is cited, in accordance with accepted academic practice. No use, distribution or reproduction is permitted which does not comply with these terms. 